\title{
Study on influencing factors and monitoring cycle of wall thickness at key locations of natural gas station
}

\author{
Wenchen Cao*, Ying Song, Liang Feng, and Shiyu Gao \\ PipeChina Southwest Pipeline Company, China
}

\begin{abstract}
In recent years, the safe and stable operation of natural gas pipeline has become the focus of the whole society. Long distance natural gas pipelines are mostly large-diameter and high pressure pipelines, so their safe operation is particularly critical. In the high-pressure natural gas pipeline transportation industry, the wall thickness of key points (elbows and tees) will be thinned due to long-term erosion of gas, resulting in serious consequences. Therefore, its wall thickness monitoring is a key work of long-distance natural gas pipeline operation units. At present, most of them adopt off-line monitoring, but the standardized monitoring cycle cannot be effectively determined, and there are no relevant standards in the industry. The author uses fluent simulation software to establish the model, and then modifies the model combined with the field data. Then, the model is used to analyze the influencing factors of key points (elbow and TEE) one by one, and select more important influencing factors to formulate the monitoring cycle of different points. The monitoring cycle can provide reference for the daily work of on-site operators, and then ensure the safe and stable operation of long-distance natural gas pipeline.
\end{abstract}

Keywords: Long distance natural gas pipeline; Influencing factors; Wall thickness; Monitoring cycle; Elbow; Tee.

\section{Introduction}

Pipeline wall thickness detection is common in oil and gas field gathering and transmission pipelines and natural gas pipelines with high water content. For the long-distance natural gas pipeline, because most of the natural gas transported is purified and there is a filter separator for water removal in each station, few people at home and abroad have studied its wall thickness, and there is also a lack of relevant standards for its wall thickness detection. Most long-distance natural gas pipelines are large-diameter and high pressure pipelines, which will have serious consequences in case of leakage. Therefore, it is necessary to study the wall thickness of key points of long-distance natural gas station.

Throughout the relevant references, the research mainly focuses on the elbow of the pipeline. Yang Decheng et al. studied the erosion law and maximum erosion position of solid particles on the lower elbow. The results show that the particle velocity has an exponential relationship with the erosion rate; The increase of pipe diameter will reduce the

* Corresponding author: caowc@pipechina.com.cn 
erosion rate of elbow, and the two show an exponential relationship; The particle diameter basically does not affect the maximum erosion position ${ }^{[1]}$; Song Xiaoqin and others studied the erosion wear failure of $90^{\circ}$ elbow in gas transmission pipeline according to the actual working conditions on site. The results show that the greater the gathering pressure is, the less the erosion wear degree of elbow is; Gravity will affect the erosion wear degree and wear area of elbow; The smaller the gathering pipe diameter and the larger the particle diameter, the more serious the erosion wear ${ }^{[2]}$; Ji Chuling et al. Simulated the erosion wear of smooth inner wall and three bionic inner wall bends: transverse groove, longitudinal groove and pit. The results show that under the same conditions, the wear resistance of the thickened elbow can be significantly improved by using the bionic surface, and the wear resistance of the thickened elbow with different surface morphology is in the order of transverse groove shape, longitudinal groove shape, pit shape and smooth shape from large to small [3]; Zheng Yunping and others used fluent to simulate the relationship between various influencing factors and the erosion position and degree of elbow, and came to the conclusion that gas erodes the inner wall of elbow in a large area, and metal chips erode locally in this area; According to the different erosion conditions of elbows at different angles, the protection idea of "fixed side, large area and emphasis" is put forward in terms of protection parts and effectiveness ${ }^{[4]}$; Hulin deeply studied the wear law and on-line monitoring of wall thickness of fluid transmission pipeline with high temperature, high pressure and high solid holdup. The results show that the wear area and wear amount of elbow are quite different with different bend diameter ratio; At the same pipe diameter, the maximum wear decreases with the increase of bending diameter ratio ${ }^{[5]}$.

The author uses fluent simulation software to establish the model, and then modifies the model combined with the field data. Then, the model is used to analyze the influencing factors of key points (elbow and TEE) one by one, and select more important influencing factors to formulate the monitoring cycle of different points. The monitoring cycle can provide reference for the daily work of on-site operators, and then ensure the safe and stable operation of long-distance natural gas pipeline.

\section{Introduction to the built model}

This paper uses the desinmodeler module of ANSYS Workbench software to draw the geometric model, the drawn elbow model and tee model.

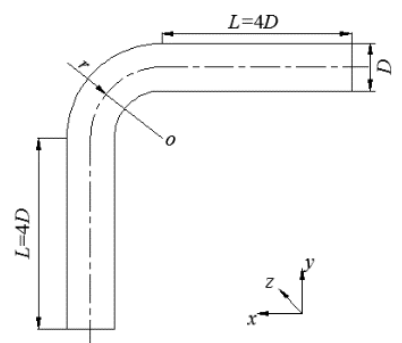

Fig. 1. Schematic diagram of elbow structure.

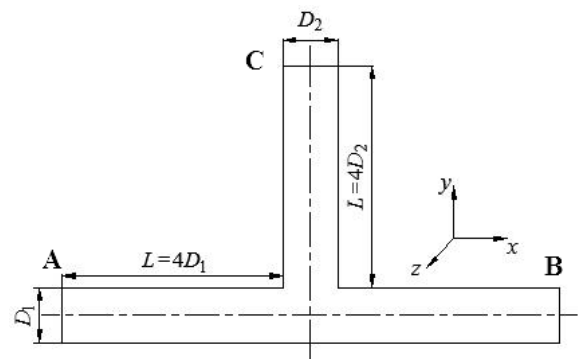

Fig. 2. Schematic diagram of tee structure.

Through the mesh module of ANSYS Workbench software, the two types of simulation models are meshed by using the proximity and curvature size function, and an expansion layer is set at the boundary to control the average quality of the grid.

During the simulation, the inlet and outlet boundary conditions are set as pressure inlet and pressure outlet. The relevant boundary conditions of fluid are set according to the parameters of specific working conditions. The gas phase material is methane, and the methane density under standard conditions is $\rho=0.714 \mathrm{~kg} / \mathrm{m}^{3}, \mu=$ one point zero eight seven 
$\times 10^{-5} \mathrm{~kg} /(\mathrm{m} \bullet \mathrm{s})$. Based on the field test data feedback model, the sand particle size is $0.4 \mathrm{~mm}$, which is close to the actual data. The particle size of the sand was used in the ancient study.

\section{Research on influencing factors}

\subsection{Influence of fluid velocity on erosion}

In the established erosion simulation model, the sand mass flow is set as $1 \times 10^{-3} \mathrm{~kg} / \mathrm{s}$, and set the fluid velocity at the inlet of the model as $5 \mathrm{~m} / \mathrm{s}, 7 \mathrm{~m} / \mathrm{s}, 9 \mathrm{~m} / \mathrm{s}, 11 \mathrm{~m} / \mathrm{s}$ and $13 \mathrm{~m} / \mathrm{s}$ respectively. The results are shown in Figure 3.

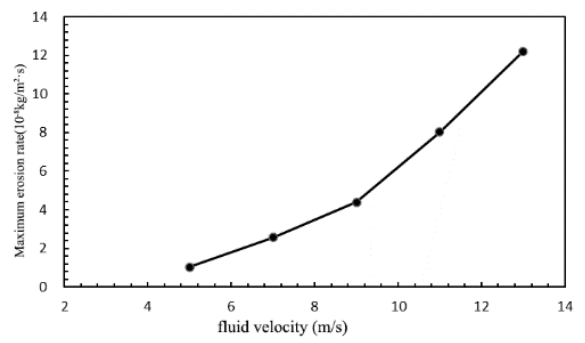

Fig. 3. Curve of fluid velocity and maximum erosion rate.

It can be seen that the maximum erosion rate at the most serious erosion point, that is, the monitoring point, increases with the increase of fluid flow rate. When the flow velocity is small, the maximum erosion rate increases slowly. When the flow velocity is large, the maximum erosion rate increases rapidly, showing an approximate exponential growth.

\subsection{Influence of pipe diameter on erosion}

In the established erosion simulation model, the mass flow rate is set as $0.02 \mathrm{~kg} / \mathrm{s}$, the inlet fluid flow rate is set as $9 \mathrm{~m} / \mathrm{s}$, and the elbow diameter of the geometric model is set as $150 \mathrm{~mm}, 200 \mathrm{~mm}, 250 \mathrm{~mm}, 300 \mathrm{~mm}, 400 \mathrm{~mm}, 500 \mathrm{~mm}$ and $900 \mathrm{~mm}$ in turn. Other parameters of the model remain unchanged. The relationship between the results obtained after simulation calculation and the maximum erosion rate is shown in Figure 4.

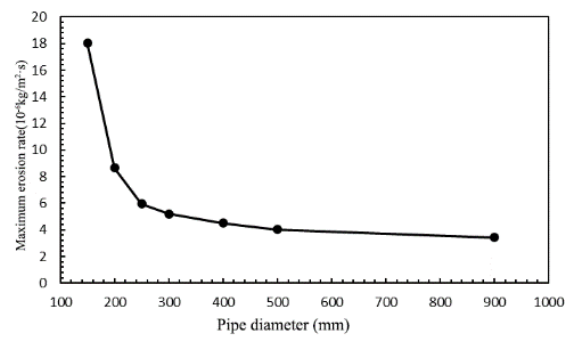

Fig. 4. Curve of pipe diameter and maximum erosion rate.

It can be seen from the figure that the erosion rate decreases rapidly with the increase of pipe diameter, and when the pipe diameter increases from $150 \mathrm{~mm}$ to $400 \mathrm{~mm}$, the erosion rate increases from $1.825 \times 10^{-5} \mathrm{~kg} / \mathrm{m}^{2} \bullet \mathrm{s}$ reduced to $4.511 \times 10^{-6} \mathrm{~kg} / \mathrm{m}^{2} \bullet \mathrm{s}$, which varies greatly. When the pipe diameter exceeds $400 \mathrm{~mm}$, the erosion rate is at a relatively low level. With the continuous increase of pipe diameter, the erosion rate decreases very slowly. 


\subsection{Influence law of sand mass flow on erosion}

In the established simulation erosion model, keep the inlet velocity of $9 \mathrm{~m} / \mathrm{s}$ unchanged, set the sand mass flow to $0.02 \mathrm{~kg} / \mathrm{s}, 0.04 \mathrm{~kg} / \mathrm{s}, 0.06 \mathrm{~kg} / \mathrm{s}, 0.08 \mathrm{~kg} / \mathrm{s}$ and $0.1 \mathrm{~kg} / \mathrm{s}$ step by step, and keep other parameters unchanged. Conduct numerical simulation on pipe models of different sizes respectively. The relationship between the maximum erosion rate and sand mass flow is shown in Figure 5.

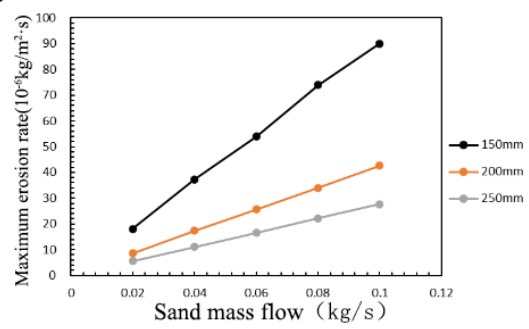

Fig. 5. Curve of sand mass flow and maximum erosion rate.

The relationship between sand mass flow and erosion rate is shown in the figure. It can be seen that with the increase of sand mass flow, the erosion rate also increases linearly. This is because with the increase of sand flow, the sand content per unit time increases and the number of collisions with pipe wall increases, resulting in the increase of pipe wall wear. It can also be concluded that with the increase of pipe diameter, the increasing speed of erosion rate with the increase of sand flow decreases gradually. Therefore, when the pipe diameter is small, the influence of sand flow on erosion rate is particularly obvious.

\subsection{Influence of bend guide on erosion}

When establishing the elbow simulation model, according to the actual installation mode of the elbow in Guangyuan gas transmission station, the simulation models of the elbow guidance as horizontal inlet, vertical upward inlet and vertical downward inlet are established, in which the first group of models are horizontal inlet and vertical downward outlet; The second group of models are vertical downward inlet and horizontal outlet; The third group of models are horizontal inlet and vertical upward outlet; The fourth group of models are vertical upward inlet and horizontal outlet; The inlet and outlet of the fifth group of models are horizontal. Other parameter settings remain unchanged. The simulation results are shown in Figure 6 and Figure 7.

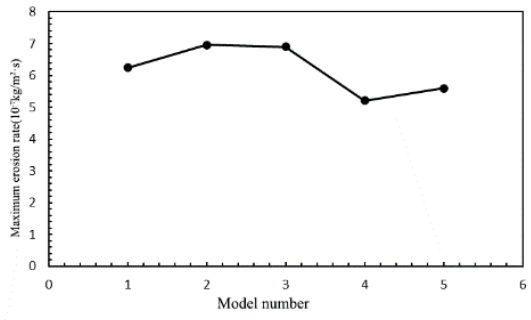

Fig. 6. Model and maximum erosion rate curve of different elbow guidance.

Figure 6 shows the impact of the inlet and outlet guidance of the elbow on the erosion rate. It can be found that the change of the erosion rate is not large. The erosion rate of the elbow is the largest under the conditions of vertical downward inlet and horizontal outlet, and the erosion rate of the elbow is the smallest under the conditions of vertical upward inlet and horizontal outlet. This is due to the increase or decrease of the incident velocity of 
sand particles under the action of gravity acceleration.

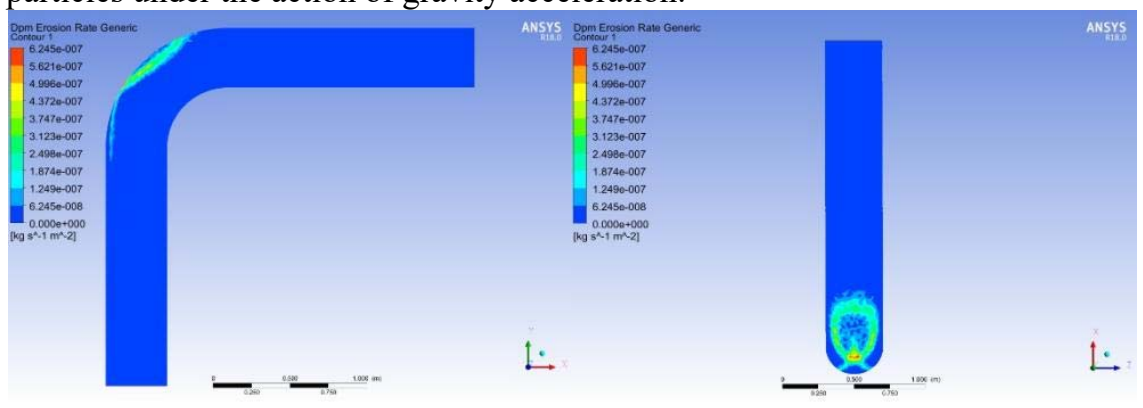

(a)

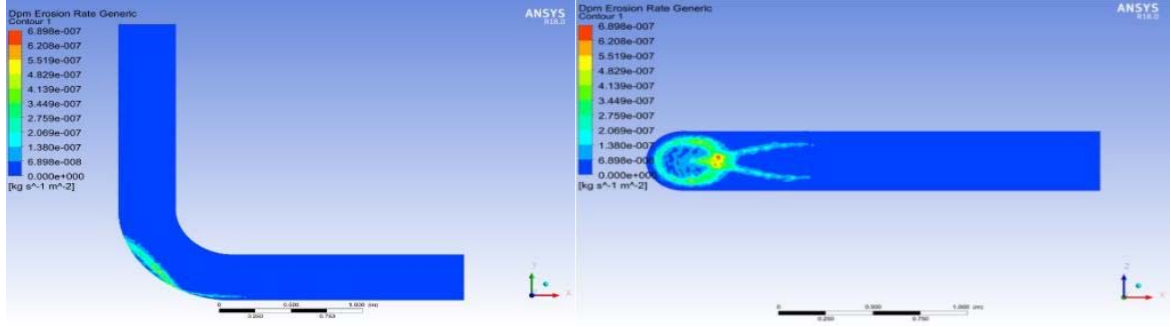

(b)

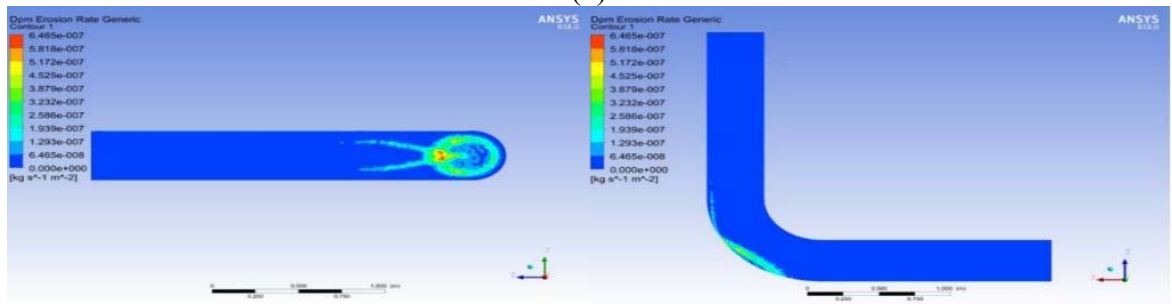

(c)

Fig. 7. (a) Elbow erosion at horizontal inlet and vertical downward outlet; (b) Elbow erosion at vertical downward inlet and horizontal outlet; (c) Elbow erosion at horizontal inlet and outlet.

It can be seen from (a-c) in Figure 7 that the elbow guide has no obvious influence on the erosion morphology, and the maximum erosion points on the elbow appear at the elbow outlet.

\subsection{Influence of bend diameter ratio on erosion}

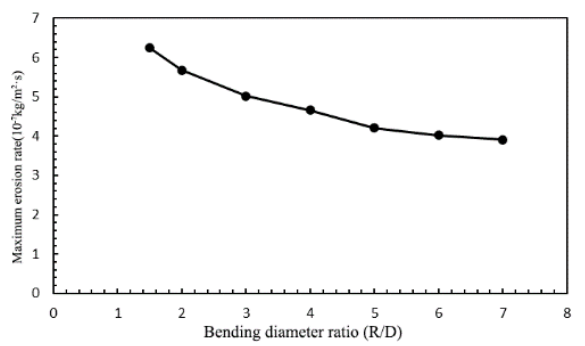

Fig. 8. Bending diameter ratio and maximum erosion rate.

When establishing the erosion simulation model, keep the inlet velocity $9 \mathrm{~m} / \mathrm{s}$ unchanged, change the bending diameter ratio $(\mathrm{R} / \mathrm{D})$ of the geometric model to 1.5, 2, 3, 4, 5, 6 and 7 
respectively, and the settings of other boundary conditions remain unchanged. The results obtained after numerical simulation are shown in Figure 8.

Figure 8 shows the influence law of the bend diameter ratio of the elbow on the erosion rate. The erosion rate increases with the increase of the bend diameter ratio, and the decreasing speed of the erosion rate will slow down with the increase of the bend diameter ratio.

\section{Determination of test cycle}

According to Q / SY 93-2007 natural gas pipeline inspection specification, the general inspection of natural gas pipeline is once a year, and the first inspection shall be carried out within half a year after putting into use. The professional inspection covering pipe wall corrosion inspection shall not exceed 8 years because it is determined according to the actual safety situation of the pipeline. The professional inspection of new pipeline shall be carried out within 3 years after putting into operation, After 3 years, the inspection cycle shall be determined according to the pipeline safety status.

In the natural gas station, combined with the research results of the previous chapter, the following table 1 is divided by pipe diameter and pressure.

Table 1. Division of inspection cycle of station pipeline.

\begin{tabular}{l|l|l|l}
\hline & less than 4MPa & less than 6Mpa & less than 10Mpa \\
\hline less than 4Mpa 300mm & Once every 4 years & Once every 3years & Once every 1 year \\
less than 4Mpa 600mm & Once every 3 years & Once every 2years & Once every 1 year \\
Not less than $600 \mathrm{~mm}$ & Once every 2 years & Once every 1 year & Once every 1 year \\
\hline
\end{tabular}

\section{Conclusion}

1. When the pipe diameter is small (below 400), the flow velocity, pipe diameter, sand flow and curvature are the main influencing factors; When the pipe diameter is larger (more than 400), the flow velocity and curvature are the main influencing factors.

2. The recommended cycle is shown in the table.

\section{References}

1. Yang Decheng, Zhu Hongwu. Erosion wear analysis of natural gas sand carrying gas-solid two-phase flow at elbow [J]. Petroleum machinery, 2019, 047 (010): 125-132

2. Song Xiaoqin, Liu Ling, Luo Songyang, et al. Study on erosion wear law of $90^{\circ}$ elbow of natural gas gathering and transmission pipeline [J]. Lubrication and sealing, 2018, 043 (008): 62-68

3. Ji Chuling. Numerical simulation of bionic wear resistance method of elbow [J]. Journal of Central South University: Natural Science Edition, 2016 (47): 3589

4. Zheng Yunping, Wang Huanhuan, Yi Haolin, et al. Simulation study on erosion and protection of natural gas pipeline elbow [J]. Computer simulation, 2015, 32 (8): $427-430$

5. Fu Lin. impact wear prediction and wall thickness monitoring of elbow of oil coal slurry transmission pipeline [D]. Hebei University of technology, 2009 